\title{
The 2000 United Kingdom Haemophilia Centre Doctors' Organisation (UKHCDO) Inhibitor Guidelines
}

\author{
C.R.M. Hay \\ Manchester Thrombosis and Haemostasis Centre, Manchester, UK
}

\section{Key Words}

Inhibitor · Guidelines · UKHCDO · Haemophilia

\begin{abstract}
The UKHCDO inhibitor guidelines address the diagnosis and management of patients with haemophilia A, haemophilia $B$ and acquired haemophilia. Recommendations are based on best current practice as reflected in the published evidence base. Many current treatment strategies are based on uncontrolled observations highlighting the need for well designed controlled studies.

Copyright $\odot 2002$ S. Karger AG, Basel
\end{abstract}

\section{Introduction}

The UKHCDO Guideline on the diagnosis and management of factor VIII and factor IX inhibitors derives from the contemporary evidence base, and represents a consensus agreed by directors of haemophilia comprehensive care centres throughout the United Kingdom [1]. The guideline provides a comprehensive and practical resource, addressing monitoring for inhibitors, laboratory diagnosis, approaches to immune tolerance induction, treatment of bleeding events and management of acquired haemophilia. Levels of evidence are those defined in AHCPR 1992 [2].

\section{Inhibitor Monitoring and Diagnosis}

The guideline recommends an APPT mixing/incubation test should be used for inhibitor screening. This is a sensitive but non-quantitative technique. The committee could not decide which of the different methods is optimal, in the absence of comparative data. Inhibitor quantification should use the Nijmegen modification of the Bethesda technique [3], as endorsed by the factor VIII and IX Scientific Subcommittee of the International Society of Thrombosis and Haemostasis [4]. Prophylactic concentrate administration should be optimized through periodic pharmacokinetic studies in individual patients. This approach incorporates some degree of surveillance for inhibitors. Often a reduced factor VIII recovery or a very low trough level during prophylaxis may be the first indicator of a low level inhibitor. The median time to development of an inhibitor is 9 to 12 exposure days [5]. For this reason inhibitor screening should be conducted initially every fifth exposure day or quarterly until the 20th exposure day, and thereafter every 6-12 months. This approach will allow early detection of low-level inhibitors, which might otherwise be missed.

\section{Immune Tolerance Induction in Haemophilia A}

Immune tolerance induction (ITI) should be considered for all newly diagnosed inhibitor patients. Such programmes should be conducted in centres with expertise

Dr. Charles Richard Morris Hay

University Department of Haematology

Manchester Royal Infirmary, Oxford Road

Manchester, M13 9RY (UK)

Tel. +44 161276 4812, Fax +44 161276 4814, E-Mail haemophilia@man.ac.uk 
and experience with this therapeutic strategy. Immune tolerance induction is a substantial financial undertaking. Interruptions to ITI are associated with increased failure rates. For this reason, realistic and appropriate funding for at least the first year should be secured in advance.

The impact of ITI on the patient and his family requires that informed consent be obtained before starting. After initial inhibitor diagnosis, the patient should not be exposed to further factor VIII until ITI begins. During this time bleeding episodes should be treated with recombinant factor VIIa (rFVIIa) until the inhibitor titre has declined to less than $10 \mathrm{BU} / \mathrm{ml}$. Studies show that the prognosis for success is improved if the inhibitor titre is lower than $10 \mathrm{BU} / \mathrm{ml}$ when ITI is commenced [6, 7]. Because tolerance induction is primarily a therapeutic strategy applied to children, it is recommended that recombinant factor VIII or IX be used, as this is consistent with the UKHCDO national guidelines and with Department of Health policy in the United Kingdom. Regardless of the inhibitor titre at the outset, most patients demonstrate an anamnestic response in the early phase of ITI, and become completely refractory to factor VIII for a period. Inhibitor bypassing therapy should be available to treat breakthrough bleeds during this time.

The optimal dose for good risk patients is unresolved and is the subject of a forthcoming international study. Poor risk patients, those with very high-level inhibitors and whose inhibitor titre is greater than $10 \mathrm{BU} / \mathrm{ml}$ when they commence ITI, are probably best tolerised using a high dose regime (greater than $100 \mathrm{IU} / \mathrm{kg}$ per day). A good risk patient is defined as under the age of five, with a starting inhibitor titre less than $10 \mathrm{BU} / \mathrm{ml}$ and an historical peak titre of less than 100 to $200 \mathrm{BU} / \mathrm{ml}[6,7]$. One of the commonest reasons to interrupt therapy is the onset of central line infection, associated with non-specific stimulation of the immune system and a rise in the inhibitor titre. Infections also lead to interruption of therapy, which may adversely influence outcome and should be avoided wherever possible [7].

\section{Treatment of Bleeding}

The choice of haemostatic product is based on the clinical bleeding severity, any historic anamnestic response, the clinical response to the various products the patient has previously been exposed to and the current inhibitor titre. Low responders have inhibitor levels of less than 5 $\mathrm{BU} / \mathrm{ml}$ and do not develop an anamnestic response on further exposure to factor VIII. High responders have his- torical inhibitor titres greater than $5 \mathrm{BU} / \mathrm{ml}$, and demonstrate an anamnestic rise in their inhibitor titre when reexposed to factor VIII.

In low responders the therapeutic options would usually be human or porcine factor VIII, rFVIIa or FEIBA. Many low responders can be maintained successfully on human factor VIII at increased dose. In the case of a high level high responding patient, human or porcine factor VIII should be reserved for life or limb-threatening bleeding events. For all other bleeding events in this patient category, rFVIIa or FEIBA are indicated. In the UK and Ireland rFVIIa is recommended as the treatment of first choice for children unresponsive to factor VIII, because of its recombinant status and favourable side-effect profile. All major bleeding events should be managed with the product most likely to produce a rapid response.

\section{Surgery}

Surgery for inhibitor patients is a high-risk procedure, and is best undertaken in an experienced centre. Human or porcine factor VIII may be used if satisfactory factor VIII levels can be achieved. In congenital as opposed to acquired haemophilia, the clinical response to porcine factor VIII correlates with the factor VIII recovery [8]. There is no conclusive evidence that rFVIIa is significantly thrombogenic. Published reports of rFVIIa use for elective surgery are accumulating. It is therefore recommended rFVIIa is first-line treatment for patients in whom a satisfactory factor VIII level cannot be achieved. There is comparatively little data on the use of FEIBA for surgery.

\section{Factor IX Inhibitors}

When considering the management of factor IX inhibitors, the central variable is whether or not they suffer from anaphylaxis. This complicates management considerably. Those with a low inhibitor titre without anaphylaxis can be successfully managed with factor IX, FEIBA or rFVIIa. High responder patients are generally refractory to factor IX, and require rFVIIa or FEIBA. National treatment policy dictates that rFVIIa is the treatment of choice for children in the UK and Ireland unresponsive to or reacting to factor IX. For minor bleeding, preference may be given to products unlikely to cause anamnesis. Recombinant FVIIa is the treatment of choice for patients who have
20

Pathophysiol Haemost Thromb 2002;32(suppl 1):19-21
Hay 
demonstrated anaphylaxis, as these reactions can be lifethreatening.

ITI for factor IX inhibitor patients is more difficult and less successful than is the case for haemophilia A. In those without anaphylaxis, high purity or recombinant factor IX is recommended to avoid the thrombotic adverse events associated with intermediate purity products. At least the first few doses should be administered in hospital in case anaphylaxis occurs. Allergic reactions arise after a median of 11 exposure days in the treatment history. Pre-medication is often only partly effective in suppressing the reactions. Patients with anaphylaxis on ITI programmes may develop nephrotic syndrome, and this condition should be monitored for [9]. This may not resolve completely when factor IX therapy is discontinued.

\section{Acquired Haemophilia}

Acquired haemophilia commonly presents from medical or surgical specialties. It is recommended experienced centres should manage these patients, particularly when bleeding complications occur. Mortality from haemorrhage has been reported in up to $22 \%$ of cases [10]. For this reason, the guideline emphasises that immunosuppressive therapy should start immediately. First line treatment should be a combination of prednisolone and cyclophosphamide. In cases of inadequate response second line treatment options include high dose immunoglobulin, cyclosporine or multiple agent chemotherapy. Immunoabsorption may be a very useful adjunct in these patients, given at the same time as the initial immunosuppression.
Human factor VIII is usually ineffective for the treatment of bleeding in acquired haemophilia. Similarly DDAVP is usually ineffective unless the inhibitor level is so low that the patient has a measurable residual factor VIII. Significant bleeding should be treated aggressively. The treatment of choice is either rFVIIa or, if available, porcine factor VIII because of the relatively low crossreactivity. Cross-reactivity as indicated by the Bethesda assay is at best semi-quantitative in this clinical context. More minor bleeding can be treated with rFVIIa, porcine factor VIII or FEIBA.

\section{Conclusion}

Most of the evidence for recommendations in this area is grade III or IV. All newly diagnosed inhibitors should be tolerised if possible. The optimal method for immune tolerance induction is disputed and trials are needed. The relative efficacy of different haemostatic treatment options are not well established and comparative studies would be very useful. Finally, effort should be directed to optimizing the contractual mechanism for funding immune tolerance induction programmes. Such consideration will inevitably involve assessment of cost-effectiveness of such therapy for different risk groups, together with analysis of risk-sharing strategies.

\section{Acknowledgement}

The inhibitor guidelines were drafted by UKHCDO inhibitor working party: CRM Hay, TP Baglin, PW Collins, FGH Hill and DM Keeling and subsequently ratified by the UKHCDO Executive Committee.

\section{References}

1 Hay CRM, Baglin TP, Collins PW, Hill FGH, Keeling DM: The diagnosis and management of factor VIII and factor IX inhibitors: A guideline from the UK haemophilia center Doctors' organization (UKHCDO). Br J Haematol 2000;111:78-90.

2 AHCPR (1992) Acute pain management: Operative or medical procedures and trauma. Agency of healthcare policy and research publications. United States Department of Health and Human Services, Washington.

3 Verbruggen B, Novakova I, Wessels H, Boezman J, van den Berg M, Mauser-Bunschoten E: The Nijmegan modification of the Bethesda assay for factor VIII:C inhibitors: Improved specificity and reliability. Thromb Haemost 1995; 73:247-250.
4 Giles A, Verbruggen B, Rivard G, Teitel J, Walker I: A detailed comparison of the performance of the standard versus the Nijmegen modification of the Bethesda assay in detecting factor VIII:C inhibitors in the haemophilia A population of Canada. Thromb Haemost 1998; 79:872-876.

5 Ehrenforth S, Kreuz W, Scharrer I, Linde R, Funk M, Gungor T, Krackhardt B, Kornhuber $B$ : Incidence of development of factor VIII and factor IX inhibitors in haemophiliacs. Lancet 1992:339:594-598.

6 DiMichele DM, Kroner BL \& the ISTH factor VIII/IX sub-committee: Analysis of the North American immune tolerance registry (NAITR)(1993-97); Current practice implications. Vox Sang 1999;77:31-32.
7 Lenk H \& the Study Group of the German Haemophilia Centres: The German National Immune Tolerance Registry, 1997 update. Vox Sang 1999;77:28-30.

8 Morrison AE, Ludlam CA, Kessler C: Use of porcine factor VIII in the treatment of patients with acquired haemophilia. Blood 1993;81: 1513-1520.

9 Wenstein BM, Takemoto C, Warrier I, Lusher J, Saidi P, Eisle J, Ettinger LJ, DiMichele DM: Nephrotic syndrome as a complication of immune tolerance in hemophilia B. Blood 1997; 89:1115-1116.

10 Hay CRM, Negrier C, Ludlam CA: The treatment of bleeding in acquired haemophilia with recombinant factor VIIa. Thromb Haemost 1997;78:1463-1467. 